\title{
ANÁLISE DE VIABILIDADE DE POPULAÇÕES: UMA FERRAMENTA PARA A CONSERVAÇÃO DA BIODIVERSIDADE NO BRASIL
}

\author{
Daniel Brito ${ }^{1,2 *}$ \\ ${ }^{1}$ Laboratório de Ecologia Aplicada e Conservação, Depto. de Ecologia, Inst. de Ciências Biológicas, Universidade Federal de Goiás (UFG). \\ Caixa Postal: 131. Goiânia, Goiás, Brasil. CEP: 74001-970. \\ ${ }^{2}$ Programa de Pós-Graduação em Ecologia e Conservação da Biodiversidade, Universidade Estadual de Santa Cruz (UESC). Ilhéus, Bahia, Brasil. \\ CEP: 45662-000. \\ E-mail: brito.dan@gmail.com (*)
}

\section{RESUMO}

A atual crise da biodiversidade é um evento de proporções globais e históricas. As taxas de extinção estão muito maiores que o esperado naturalmente, e esta tendência pode ser particularmente preocupante para países com níveis elevados de biodiversidade, como o Brasil. A Análise de Viabilidade de Populações (AVP) é um conceito relativamente recente, e técnicas associadas a ele têm sido desenvolvidas para auxiliar a conservação a atingir o objetivo de manter populações viáveis em longo prazo. Conforme as populações se tornam pequenas, processos estocásticos (estocasticidade demográfica, estocasticidade ambiental, estocasticidade genética, perda de flexibilidade evolutiva, instabilidade metapopulacional e ruptura de estrutura social) podem desestabilizar as populações, levando-as à extinção. Estes processos podem ser examinados em modelos detalhados de AVP, que também auxiliam fornecendo direcionamento para diversos aspectos de avaliações (aonde o objetivo é simplesmente perguntar o quão saudável é a população) e manejo (onde o objetivo é determinar quais intervenções vão reduzir a probabilidade de extinção), auxiliando a biologia da conservação a caminhar em direção ao objetivo final de preservar populações viáveis. Comparado com outros países, como Estados Unidos e Austrália, o Brasil ainda está relativamente atrasado em desenvolver pesquisas com AVP, mas parece haver uma tendência para crescimento no seu uso nos anos recentes. Entretanto algumas preocupações, envolvendo representação taxonômica e geográfica da técnica dentro do país e a publicação formal dos resultados das pesquisas (muitas AVPs no Brasil são publicadas na literatura cinza), ainda precisam ser abordadas e solucionadas. A AVP pode ajudar não apenas biólogos, mas também tomadores de decisão, ajudando a sociedade brasileira atingir o objetivo de avaliar, monitorar e assegurar a viabilidade a longo prazo da biodiversidade nacional. Em função disso, seu uso e disseminação, associados a capacitação dos biólogos da conservação brasileiros em conduzir e liderar tais análises devem estar entre as prioridades de conservação no país.

Palavras-chave: análise de viabilidade populacional, estocasticidade, extinção, populações pequenas

\begin{abstract}
POPULATION VIABILITY ANALYSIS: A TOOL FOR BIODIVERSITY CONSERVATION IN

BRAZIL. The current biodiversity crisis is an event of global and historical proportions. Extinction rates are much higher than natural expected rates, and this trend may be particularly worrisome for countries with high levels of biodiversity, like Brazil. Population viability analysis (PVA) is a relatively new concept and technique, developed to help achieve the conservation goal of maintaining viable populations in the long-term. As populations become smaller, interacting stochastic processes (demographic stochasticity, environmental stochasticity, geetic stochasticity, loss of adaptive flexibility, metapopulation instability and social system disruption) can destabilize populations and lead them to extinction. These processes can be examined in detailed PVA models, which can also provide insights and guidance in several aspects of assessments (in
\end{abstract}


which the goal is simply to ask how well the population is doing) and management (in which the goal is to determine what interventions will reduce the population's likelihood of extinction), helping conservation Biology move towards the ultimate goal of preserving health viable populations. Comparing to other countries, like Australia and the United States, Brazil still lags behind in developing PVA research, but there seems to be a tendency for a slight increase in its use in recent years. However a few concerns regarding proper geographic and taxonomic coverage within Brazil, and the formal publication of research results (many PVAs in Brazil are published in the grey literature) still need to be addressed and have room for improvement. PVA can help not only Brazilian conservation biologists and managers, but also decision and policy makers in achieving the ultimate goal of assessing, monitoring and ensuring long-term viability for the country's biodiversity and its use, coupled with building the capacity for Brazilian conservation biologists to lead such analyses, should be among the priorities within Brazil.

Keywords: extinction, population viability analysis, small populations, stochasticity

\section{RESUMEN}

ANALISIS DE LA VIABILIDAD DE LAS POBLACIONES: UNA HERRAMIENTA PARA LA CONSERVACIÓN DE LA BIODIVERSIDAD EN BRASIL. La actual crisis de biodiversidad es un evento de proporciones globales e históricas. Las tasas de extinción son mayores que lo esperado naturalmente, y esta tendencia puede ser particularmente preocupante para países con niveles elevados de biodiversidad como Brasil. El Análisis de Viabilidad de Poblaciones (AVP) es un concepto relativamente reciente, las técnicas asociadas a él han sido desarrolladas para apoyar a las iniciativas de conservación a alcanzar el objetivo de mantener la viabilidad de las poblaciones a largo plazo. A medida que las poblaciones se tornan pequeñas, procesos estocásticos (estocasticidad demográfica, estocasticidad ambiental, estocasticidad genética, pérdida de flexibilidad evolutiva, inestabilidad metapoblacional y ruptura de estructura social) pueden desestabilizar a las poblaciones, llevándolas a la extinción. Estos proceso pueden ser examinados en modelos detallados de AVP, que también proporcionan direccionamientos para diversos aspectos de evaluaciones (donde el objetivo es simplemente preguntar cuán saludable es la población) y manejo (donde el objetivo es determinar cuáles intervenciones van a reducir la probabilidad de extinción), ayudando a la Biología de la Conservación a alcanzar el objetivo final de preservar las poblaciones viables. Comparado con otros países como Estados Unidos ó Australia, Brasil aún está relativamente atrasado en desarrollar investigaciones con AVP, aunque en los últimos años, parece haber una tendencia al crecimiento en su utilización. Sin embargo algunas preocupaciones, incluyendo la representación taxonómica y geográfica de la técnica dentro del país y la publicación formal de los resultados de las investigaciones (numerosas AVPs de Brasil han sido publicadas en literatura gris), aún precisan ser abordadas y solucionadas. La AVP puede ayudar no sólo a los biólogos, sino también a las personas encargadas de tomar decisiones, ayudando a la sociedad brasilera a alcanzar el objetivo de evaluar, monitorear y asegurar la viabilidad a largo plazo de la biodiversidad nacional. En función de esto, su uso y difusión, asociado con la capacidad de los biólogos de la conservación brasileros en conducir y liderar tales análisis deben estar entre las prioridades de conservación del país.

Palabras-clave: Análisis de viabilidad poblacional, estocasticidad, extinción, poblaciones pequeñas

\section{INTRODUÇÃO}

A atual crise da biodiversidade é um evento de proporções globais e históricas (Wilson 1988, Pimm \& Brooks 1997, Baillie et al. 2004). Impactos de origem antrópica aumentaram as taxas de extinção para níveis entre 100 e 1000 vezes maiores que as taxas naturais (Pimm \& Brooks 1997, Baillie et al.
2004). Nas regiões tropicais, entre $0,1 \%$ e $0,3 \%$ das espécies, e $0,8 \%$ das populações se extinguem a cada ano (Balmford et al. 1998). Esta perda é evidente e preocupante no Brasil, um dos países megadiversos do mundo (Mittermeier et al. 1997), onde estimativas indicam que o país deve conter aproximadamente $13 \%$ da biodiversidade global (Lewinsohn \& Prado 2005). Se queremos alcançar a conservação da 
biodiversidade frente a crescente demanda de exploração por recursos naturais no Brasil, a viabilidade de populações silvestres, de processos ecológicos e evolutivos e dos serviços de ecossistemas devem ser considerados nos planos e ações de manejo e na elaboração de políticas públicas.

A questão sobre quais são as condições mínimas para a persistência a longo prazo e adaptação de uma espécie e/ou população em um determinado local éuma dos mais difíceis e desafiadores problemas em biologia da conservação (Soulé 1987). A Análise de Viabilidade de Populações (AVP) representa um novo conceito e uma nova gama de técnicas, úteis para se atingir tal objetivo. A AVP é uma metodologia para predizer o futuro estado de populações silvestres baseada em parâmetros demográficos, ambientais e genéticos (Shaffer 1981, Gilpin \& Soulé 1986, Boyce 1992, Burgman et al. 1993, Norton 1995), mais comumente através do uso de modelagem computacional. $\mathrm{O}$ processo da AVP incita uma importante abordagem interdisciplinar, além de sintetizar o estado do conhecimento sobre a espécie e/ou área de estudo, sendo uma ferramenta influente e amplamente aplicada em biologia da conservação. A AVP pode ser usada para comparar estratégias de manejo de acordo com seu impacto relativo na persistência de populações silvestres, comparar ameaças de acordo com seu impacto relativo nas populações, projetar tendências populacionais, avaliar risco de extinção, guiar decisões de políticas ambientais, e auxiliar no design e efetividade de sistemas de áreas protegidas (Clark et al. 1991, Boyce 1992, Lindenmayer et al. 1993, Beissinger \& Westphal 1998, Morris \& Doak 2002). O Conservation Breeding Specialist Group (CBSG) da World Conservation Union (IUCN) já realizou mais de 130 AVPs (CBSG 2009) e muitas mais também já foram feitas (e.g. Lindenmayer \& Possingham 1994, Brook et al. 1999). Os resultados das AVPs podem ser usados para categorizer o grau de ameaça e dar suporte às conclusões atingidas com base nos critérios da IUCN (IUCN 2001).

Algumas críticas foram levantadas sobre o uso e a utilidade da AVP para a biologia da conservação, tanto como ferramenta de manejo quanto ao seu embasamento teórico (Ralls \& Taylor 1997, Coulson et al. 2001, Ellner et al. 2002). Entretanto, a maioria das críticas não reflete fraquezas da AVP em si, mas sim limitações e usos errados da técnica. É importante notar que quando a AVP surgiu, ela era vista quase como o "cálice sagrado" da biologia da conservação, e uma perspectiva ingênua que ela traria soluções para a maioria das questões de conservação rapidamente se desenvolveu (Brito \& Fernandez 2000a). A AVP é uma ferramenta útil e robusta, mas como qualquer ferramenta, ela possui pontos fortes, fracos e limitações (Lindenmayer et al. 1993). Isto não significa que ela seja inútil, apenas significa que como em qualquer técnica, nós devemos levar em consideração todos estes fatores ao conduzir este tipo de análise. A AVP já provou sua utilidade como ferramenta para guiar o manejo de populações (Boyce 1992, Lindenmayer et al. 1993, Possingham et al. 1993, 2001, Reed et al. 1998, Brook et al. 2000, 2002), e é a melhor ferramenta que temos até o momento para abordar estas questões (Brook et al. 2000, 2002). Frente a atual crise de extinções, nós não temos o luxo de esperar para tomar ações, pois as consequências são a extirpação de muitas espécies (Soulé 1985). Em disciplinas de crises, como a biologia da conservação, deve-se agir antes de conhecer todos os fatos, e um biólogo da conservação deve estar preparado para tomar decisões, ou fazer recomendações, antes mesmo que ele esteja completamente confortável com as bases teóricas e empíricas das análises (Soulé 1985). A AVP também promove uma filosofia preventiva de manejo, pois além de abordar os problemas relacionados com espécies ameaçadas, também devemos manter em mente que é melhor proteger as espécies antes que elas se tornem ameaçadas (Conway 1995). Não é surpresa que a AVP tenha se tornado um dos baluartes da biologia da conservação, e que o conceito de viabilidade tenha contribuído tanto para a teoria quanto para a prática em conservação, pois a AVP permitiu a formação de uma estrutra teórica e prática para estudar e compreender como eventos e processos estocásticos afetam a probabilidade de extinção (Lande 2002).

\section{OS DOIS PARADIGMAS DA BIOLOGIA DA CONSERVAÇÃO}

O papel da estocasticidade na extinção tem sido chamado de o "paradigma das populações pequenas" (Caughley 1994). Poucos pesquisadores negam a noção que processos estocásticos podem resultar em extinção, apesar que o peso relativo de cada um dos processos ainda é fonte de debates (Lande 1988, 
1993, Allendorf \& Ryman 2002). Os processos que fazem uma população declinar são chamados de o "paradigma das populações em declínio" (Caughley 1994). Ele consiste em determinar se há uma tendência populacional, identificar quais os elementos da demografia de um organismo estão deprimidos, determinar os fatores ambientais e/ou intrínsecos que causam a depressão no parâmetro demográfico, e então examinar o que pode ser feito para corrigir estes fatores limitantes. Neste paradigma a estocasticidade raramente é identificada como uma causa específica de baixa taxa reprodutiva ou de sobrevivência. Hedrick et al. (1996) argumentam que os processos que causam declínios populacionais e os problemas enfrentados por populações pequenas estão interligados de maneiras tão complexas que as abordagens em biologia da conservação, e consequentemente a AVP, devem abranger os dois paradigmas para terem sucesso em preservar a biodiversidade. Não é surpresa que na verdade, os dois paradigmas oferecem maneiras complementares de se analisar um mesmo problema (Brito \& Fernandez 2000a).

\section{UMA BREVE HISTÓRIA DA AVP}

Em função de ser relativamente recente (Soulé 1987), a AVP é extremamente dinâmica. Nas fases iniciais de seu desenvolvimento, a ciência da biologia da conservação sofria de reducionismo. A predição e o desenvolvimento de estratégias para evitar extinções eram tema central da disciplina. Ecólogos de comunidades focavam em áreas mínimas para a viabilidade de sistemas, com contribuições da biogeografia de ilhas, enquanto que ecólogos de populações focavam em tamanhos ou densidades populacionais mínimas (Soulé 1987). A dificuldade se dava pois o tópico de extinções era abordado unidimensionalmente por várias perspectivas disciplinares (Soulé 1987): genética de populações, biogeografia de ilhas, dinâmica populacional estocástica (Soulé 1987, Gilpin 1996). Portanto, nós tinhamos respostas disciplinares. Baseado em modelos de estocasticidade demográfica, estimativas sugeriam que 10 indivíduos eram necessários para tornar uma população segura frente a flutuações aleatórias nas taxas de natalidade e mortalidade (MacArthur \& Wilson 1967). A genética de populações estabeleceu dois limites: uma população menor que 50 indivíduos se extingue em curto prazo devido à depressão endogâmica, e uma populacão menor que 500 indivíduos perderia flexibilidade evolutiva e se extinguiria em face a mudanças ambientais (Franklin 1980). A biogeografia de ilhas também ofereceu estratégias, como por exemplo o debate sobre SLOSS (single large or several small) (Diamond 1975).

Shaffer (1981) desenvolveu pela primeira vez, um modelo estocástico de simulação populacional que incorporava eventos aleatórios, especificamente estocasticidades demográfica e ambiental. Este modelo produziu estimativas de probabilidade de extinção e de tamanhos de população mínima viável (PMV), ao variar o número inicial de indivíduos para encontrar o menor tamanho populacional que apresentasse 95\% de chance de persistência em um período de 100 anos (Shaffer \& Samson 1985). Além disso, pela primeira vez abordagens genéticas para avaliar a viabilidade de populações foram compiladas em uma única técnica (Frankel \& Soulé 1981). Os efeitos a curto e longo prazo da endogamia e da deriva gênica foram colocados em uma estrutura metodológica que clarificava seus impactos negativos na diversidade genética. Foi aqui onde a regra de 50/500 para tamanhos efetivos de populações (Franklin 1980, Soulé 1980) foi descrita de forma que se tornou, para bem ou para mal, uma das poucas regras na teoria de viabilidade. Logo artigos estimando PMV começaram a aparecer (e.g. Lacava \& Hughes 1984, Lehmkuhl 1984, Shaffer \& Samson 1985, Menges 1986, Reed et al. 1988). Entretanto, um erro comum é esquecer que estes "números mágicos" são apenas guias, e começar a vê-los como verdades imutáveis. O passo para o desenvolvimento da AVP à partir da PMV aconteceu quando biólogos australianos estavam preocupados em gastar seus parcos recursos financeiros de conservação no manejo e monitoramento de uma espécie que havia declinado para 48 indivíduos, um caso fadado à extinção de acordo com a regra 50/500 (Gilpin 1996). É importante lembrar que em processos onde estocasticidade está envolvida, não podem haver números mágicos ou regras absolutas. Seria ingênuo imaginar que um único valor teria validade universal frente a grande diversidade biológica (Soulé 1987). Para resolver esta questão, Gilpin \& Soulé (1986) criaram um conceito heurístico para examinar as diversas forças que interagem e que podem afetar a viabilidade de uma população, inclusive fatores genéticos. Assim nasceu a AVP. A idéia da AVP 
como um processo de análise de risco emergiu, onde as ameaças são identificadas, os riscos considerados e um modelo é desenvolvido.

As aplicações da AVP cresceram rapidamente, e nos últimos 20 anos, os modelos se tornaram mais complexos, incorporando processos espaciais. Os modelos também passaram de populacões únicas para modelos metapopulacionais, primeiro tratando a demografia no nível de manchas homogêneas, depois amadureceram para modelos em grid espacialmente explícitos com manchas heterogêneas e finalmente atingindo a tendência atual de modelos baseados no indivíduo, que acompanham a trajetória de vida de cada indivíduo da população, enquanto ele vive, dispersa pela paisagem, se reproduz e morre (Beissinger \& Westphal 1998).

\section{POPULAÇÃO MÍNIMA VIÁVEL (PMV)}

A abordagem da PMV procura determiner o número mínimo de indivíduos que uma população precisa ter para persistir por um certo período de tempo (Shaffer 1981, Gilpin \& Soulé 1986, Soulé 1987). O conhecimento sobre tamanho populacional é tão importante pois populações pequenas têem mais chances de se extinguir que populações grandes (Pimm et al. 1988, Caughley \& Gunn 1996). Portanto, estimar PMVs é um aspecto fundamental em biologia da conservação (Shaffer 1981, Belovsky 1987). Franklin (1980) propôs que o tamanho efetivo da populacão $(\mathrm{Ne})$ não deve ser menor que 50 para assegurar sobrevivência em curto prazo, e que um $\mathrm{Ne}$ de 500 seria necessário para garantir persistência a longo prazo. Juntas, as estimativas de PMV para persistência em curto e longo prazo resultaram na conhecida regra 50/500, que foi amplamente usado como objetivo de manejo para um grande número de espécies ameaçadas (Lande \& Barrowclough 1987). Entretanto, pesquisas recentes sugerem que estes tamanhos populacionais provavelmente subestimam o tamanho de PMV para diversas populações por várias razões, e que a PMV pode ser consideravelmente maior que 500 (Lande 1995, Lynch \& Lande 1998, Reed \& Bryant 2000). Proteger a biodiversidade reuqer a proteção de populações saudáveis de todas as espécies, e para proteger as espécies é necessário habitat suficiente para comportar PMVs (Allen et al. 2001). Entretanto, logo se tornou óbvio que não existe um único "número mágico" que seja universalmente válido (Soulé 1987). Um aspecto importante do conceito, que é algumas vezes esquecido, faz toda a diferença. Como os processos ameaçando populações pequenas são estocásticos, a definição de PMV deve ser probabilística ao invés de determinística (Shaffer 1981, Brito \& Fernandez 2000a). A PMV então, é definida como o número mínimo de indivíduos que uma população precisa ter para assegurar que ela possua uma certa probabilidade de sobrevivência em um certo período de tempo (por exemplo, 95\% de chance de persistência em 100 anos). As estimativas de PMV podem variar caso a caso, por exemplo de acordo com objetivos de conservação, grau de segurança ou as condições iniciais do cenário.

\section{PROCESSOS ESTOCÁSTICOS E VIABILIDADE POPULACIONAL}

Existem diversos tipos de ameaças à viabilidade de populações. Alguns processos levam populações grandes e contínuas a se tornarem menores e subdivididas, como por exemplo exploração, introdução de espécies exóticas, e perda e fragmentação de habitats (Caughley 1994). Conforme as populações se tornam menores, ameaças adicionais à sua persistência surgem, que podem exarcebar a dificuldade em reverter ou parar um declínio. Os problemas de pequenas populações geralmente resultam de processos estocásticos. Em qualquer processo de amostragem, a previsibilidade do resultado decresce conforme o tamanho da amostra é reduzido (Lacy 2000). Muitos aspectos de dinâmica populacional são inerentemente processos de amostragem, ao invés de eventos determinísticos. A incerteza em tais processos pode levar à instabilidade na dinâmica populacional. Reduções nas taxas de crescimento e flutuações nas taxas podem interagir, aumentando a instabilidade e causando declínios, até que a estabilidade é alcançada quando a população se extingue. Estes processos foram denominados vórtices de extinção (Gilpin \& Soulé 1986) e seu estudo constitui a base da maioria das AVPs (Soulé 1987, Boyce 1992, Lacy 1993,1994). A seguir, é apresentada uma breve revisão dos processos estocásticos que ameaçam a viabilidade de populações pequenas. Apesar de ser didático apresentar cada processo estocástico separadamente, na prática eles em geral ocorrem concomitantemente (Gilpin \& Soulé 1986, Lacy 1997, 2000, Brito \& Fernandez 2000a). 


\section{ESTOCASTICIDADE DEMOGRÁFICA}

A estocasticidade demográfica é a variação aleatória nos números de nascimentos, mortes, estrutura etária e razão sexual em uma população, resultante do fato que o destino dos indivíduos são resultados independentes de eventos probabilísticos de reprodução, sobrevivência e determinação sexual (Shaffer 1981). Todas as variações na estrutura demográfica de uma população (afetando as taxas de natalidade, mortalidade, imigração e emigração), vão afetar as taxas de crescimento populacionais. A flutuação nas taxas de crescimento ao longo do tempo podem ser tão importantes para a persistência da população quanto a magnitude da taxa em si (Brito \& Fernandez 2000a). Populações com flutuações maiores na taxa de crescimento apresentam menor tempo de persistência pois baixas taxas de crescimento quando a população está num vale da flutuação fazem com que ela demore mais tempo para se recuperar destes tamanhos populacionais pequenos, e portanto ela fica exposta mais tempo aos riscos associados a pequenos tamanhos (Belovsky 1987, Nunney \& Campbell 1993).

A estocasticidade demográfica é reconhecida como uma ameaça potencial para populações pequenas, mas a contribuição que ela exerce para a instabilidade populacional tem sido subestimada (Lacy 2000). A probabilidade que todos os indivíduos de uma população sejam do mesmo sexo, ou morram sincronicamente, pode causar extinções e afeta a viabilidade de populações pequenas (Goodman 1987, Shaffer 1987). Entretanto, problemas que não são ameaças sérias sozinhos, podem se tornar contribuintes importantes para a o declínio e instabilidade populacional quando agem em conjunto com outras ameaças. Por exemplo, a probabilidade que uma população com 10 indivíduos reprodutores produza filhotes todos do mesmo sexo em uma determinada estação reprodutiva é de aproximadamente $3 \%$, enquanto que em uma população com 100 indivíduos esta probabilidade é praticamente zero, podendo-se assumir que flutuações aleatórias na razão sexual não são importantes em populações grandes. Entretanto, tais flutuações podem deprimir a taxa de crescimento significativamente mesmo em tais casos. Numa população com 100 indivíduos reprodutores, espera-se que hajam 50 machos e 50 fêmeas, mas a estocasticidade demográfica vai desviar a razão sexual em 5 ou mais indiví- duos (1 DP) do esperado em aproximadamente 1/3 dos anos (Lacy 2000). Em espécies monogâmicas, isto significa que menos que 50 pares reprodutivos podem ser formados. Devido exclusivamente à flutuações na razão sexual, a reprodução em uma população monogâmica com 100 adultos pode ser deprimida em aproximadamente 8\% (Lacy 2000). Este nível de redução na produtividade populacional é suficiente para levar espécies com baixa fecundidade de uma taxa de crescimento positiva para negativa. A interação do sistema reprodutivo com flutuações na razão sexual podem influenciar o crescimento populacional (Brook et al. 1999, Lacy 2000). Desvios aleatórios na razão sexual podem causar extinção em populações pequenas, mas também podem interagir com outros fatores, como o sistema reprodutivo, e deprimir o crescimento populacional mesmo em populações relativamente grandes. Muitos modelos populacionais ignoram completamente razão sexual e sistema reprodutivo, projetando apenas o número de fêmeas sobre a premissa de que sempre haverão machos disponíveis para reproduzir (Lacy 2000), e isto pode levar a uma subestimativa dos riscos de declínio populacional e extinção.

De maneira similar, estocasticidade demográfica afetando os números de nascimentos e mortes pode deprimir o crescimento populacional devido à variações na estrutura etária e outras influências em reprodução (Lacy 2000).

\section{ESTOCASTICIDADE AMBIENTAL}

Estocasticidade ambiental; é a variação em taxas demográficas resultantes de flutuações naturais do ambiente (Shaffer 1981, Lacy 2000). Variações ambientais locais causam diferenças temporais nos nascimentos e mortes, o que pode aumentar a incerteza e a variabilidade no tamanho populacional, tornando uma populacão pequena mais vulnerável à extinção. Os tipos de perturbações ambientais que podem causar variações nas taxas de natalidade e mortalidade incluem disponibilidade irregular de recursos, clima variável, entre outros, por exemlo. Catástrofes naturais são o extremo da variação ambiental, e incluem secas, enchentes, incêndios, epidemias, tempestades e outros desastres locais que podem dizimar uma população (Brito \& Fernandez 2000a, Lacy 2000). 
Apesar de tanto a estocasticidade demográfica quanto a ambiental causarem flutuações nos números de nascimentos e mortes em uma população, os processos são conceitualmente distintos. A estocasticidade demográfica é intrínsica à todas as populações, independente do grau de estabilidade do ambiente, e é altamente dependente do tamanho populacional (denso-dependente). A estocasticidade ambiental resulta de variações na qualidade do habitat ao longo do tempo, e não está relacionada com o tamanho populacional (denso-independente). A variância nas taxas demográficas causada por estocasticidade ambiental será aditiva à variância devido à estocasticidade demográfica (Goodman 1987). Apesar da variação ambiental não ser geralmente afetada pelo tamanho local da população, a ameaça à viabilidade populacional será mais severa em populações menores, pois populações menores estão mais perto da extinção. Além disso, a quantidade de variação ambiental deve ser altamente dependente da área total de habitat ocupado por uma população. Muitos estresses ambientais são localizados, portanto, uma população ocupando uma grande área se beneficiaria do balanço (em média) de qualquer flutuação ambiental que não seja sincrônica ao longo de toda sua área de distribuição. Indivíduos podem usar a heterogeneidade espacial do habitat para escapar de variações temporais no ambiente (Kindvall 1996). Mesmo se os indivíduos não se moverem para escapar de áreas com condições temporariamente inadequadas, declínios populacionais temporários nesta localidade seriam balanceados por crescimento positivo em outras regiões da área de distribuição.

\section{ESTOCASTICIDADE GENÉTICA}

Existem diversos tipos de problemas genéticos que podem impactar a viabilidade de populações pequenas, sendo os principais a redução de aptidão devido à depressão endogâmica e a perda de diversidade genética devido à deriva gênica (Lacy 2000).

Depressão endogâmica, a redução na aptidão de indivíduos endocruzados, ocorre quando organismos reproduzem com parentes próximos. Este processo é um problema pode ser persistente ou transitório, diminuindo conforme a seleção natural remove alelos deletérios ao longo de gerações sujeitas ao endocruzamento (Lacy 2000). Um número crescente de estudos demonstram que a depressão endogâmica pode impactar a viabilidade populacional de forma mais séria e rápida que se supunha previamente (Frankham 1995, Lacy 1997). Alguns autores acreditam que uma taxa lenta de endocruzamento não vai reduzir a aptidão, pois a seleção natural pode remover os alelos deletérios durante o tempo que a população está sujeita ao endocruzamento (Charlesworth \& Charlesworth 1987). Entretanto, evidências empíricas sugerem que o purgamento da carga genética de alelos deletérios de uma população nem sempre acontece, e muitas populações continuam a apresentar declínio na aptidão conforme elas se tornam mais endocruzadas (Ballou 1997, Lacy \& Ballou 1998), e podem se extinguir como consequência (Frankham 1995). Trabalhos teóricos indicam porque a seleção natural é muitas vezes ineficiente em reduzir a depressão endogâmica. Em tamanhos populacionais pequenos, a deriva gênica é uma força muito mais forte em determinar quais alelos aumentam ou diminuem de frequência do que a seleção natural. A perda aleatória de alelos adaptativos é praticamente tão provável de ocorrer quanto a perda de alelos deletérios. Exceto quando a depressão endogâmica ocorre primariamente devido a poucos alelos recessivos altamente deletérios, a endogamia possui maior probabilidade de resultar em extinção do que na redução significativa da carga genética (Hedrick 1994). Algumas populações podem ter a sorte de não apresentar uma carga genética de alelos deletérios que seriam expressos sob endocruzamento, mas evidências sugerem que a sensibilidade ao endocruzamento pode ser determinada tanto por eventos ao acaso, tais como efeito fundador, como por qualquer fator previsível (Ralls et al. 1988, Lacy et al. 1996). Mutações deletérias podem ser fixadas em populações pequenas devido à deriva, um processo conhecido como mutational meltdown. Conforme tais alelos vão se acumulando, o tamanho populacional declina mais rapidamente, fazendo com que a deriva gênica proceda ainda mais intensamente (Brito \& Fernandez 2000a). Hibridização e introgressão são outros problemas genéticos sérios que ameaçam populações pequenas. Estes processos geralmente estão relacionados com modificação de habitats e/ou introdução de espécies exóticas, e o perigo é a extinção genética, ou seja, a completa assimilação genética de uma espécie rara por outra comum (Rhymer \& Simberloff 1996, Simberloff 1996). 


\section{PERDA DE FLEXIBILIDADE EVOLUTIVA}

Espera-se que a taxa de evolução adaptativa de qualquer população seja proporcional à sua diversidade genética para as características que estejam sob seleção. Este processo refere-se a perda da habilidade de uma população em responder adaptativamente à mudanças ambientais futuras devido à perda de variabilidade genética, que é a matéria prima usada pela seleção natural para exercer seu papel (Gilpin \& Soulé 1986, Brito \& Fernandez 2000a, Lacy 2000). Devido a atual situação de mudanças ambientais sem precedentes, e uma acelerada taxa de extinção, modelos de AVP deveriam considerar a manutenção de diversidade genética suficiente para assegurar flexibilidade ecológica e evolutiva, ao invés de focar exclusivamente na aptidão imediata e em persistência a curto prazo. Uma maneira de acomodar viabilidade de longo prazo no planejamento de conservação seria usar o potencial de recuperação ao invés de simplesmente a persistência populacional em tamanhos pequenos (Lacy 2000). Esta é uma ameça séria, mas muitas vezes subestimada. Como a hipótese da Rainha Vermelha ressalta, uma população deve evoluir continuamente para sobreviver (van Valen 1973).

\section{INSTABILIDADE METAPOPULACIONAL}

Para espécies naturalmente estruturadas em metapopulações, ou no caso de espécies que se estruturam em metpopulações como consequência de perda e fragmentção de habitats, a conectividade é uma parâmetro de importância fundamental para se avaliar persistência. Dispersão pode ser um assunto controverso (Clinchy 1997). Acredita-se que um aumento de dispersão entre populações geralmente ajuda a estabilizar uma metapopulação. Um aumento na dispersão pode restaurar variabilidade genética em populações que tenham passado por depressão endogâmica, pode reduzir flutuações demográficas em populações locais, pode resgatar populações demograficamente frágeis (Brown \& Kodrick-Brown 1977), e pode levar à recolonização de populações locais temporariamente extirpadas. Entretanto, a dinâmica metapopulacional de populações pequenas, parcialmente isoladas e frequentemente extirpadas, pode ser altamente dependente de aspectos espaciais, temporais e comportamentais da estrutura populacional (Fahrig \&
Merriam 1994). Por exemplo, se uma metapopulação declina a um nível em que muitas das suas populações constituintes seja muito pequenas ou estejam extintas, os benefícios da dispersão podem ser suplantados por suas desvantagens. Emigração não-compensada de populações isoladas pode deprimir o crescimento populacional local (Fahrig \& Merriam 1985), e habitats adequados que estejam temporariamente vazios podem atuar como "escoadouros" (sinks) onde indivíduos falham em encontrar parceiros (Gyllenberg \& Hanski 1992, Brito \& Fernandez 2002). Consequentemente, um aumento na dispersão pode acelerar o declínio de metapopulações esparsamente povoadas (Lindenmayer \& Lacy 1995). Portanto, mesmo que tenha sido sugerido que um aumento nas taxas de dispersão possa aumentar a probabilidade de persistência quando a metapopulação é composta por populações relativamente grandes, em alguns casos a dispersão pode ser detrimental para a persistência, se as populações forem pequenas (Lindenmayer \& Lacy 1995). Estas evidências sugerem que em populações muito pequenas, que já são demograficamente instáveis e estão mais susceptíveis à estocasticidade demográfica, uma maior conectividade entre manchas de habitat pode desestabilizar ainda mais a dinâmica metapopulacional e contribuir para um aumento do risco de extinção. Este padrão pode ser resultado de processos de retroalimentação negativa, criando um vórtice de extinção metapopulacional (Lindenmayer \& Lacy 1995). Tais fatores devem ser levados em consideração ao se planejar estratégias de manejo visando a persistência de populações, quando frequentemente um aumento na conectividade é considerado como benéfico a priori, para a viabilidade a longo prazo (Brito \& Fernandez 2000a).

Este colapso em um vórtice metapopulacional pode ser mais provável se o comportamento de dispersão evoluiu em um ambiente diferente das condições atuais. Por exemplo, em hábitats grandes e contínuos, o padrão ótimo de dispersão pode ser subadultos dispersando em direções aleatórias para estabelecer novos territórios. Quando o habitat é altamente fragmentado, pode ser mais adaptativo permanecer no sítio natal a menos que a densidade local se torne muito alta (Ronce et al. 2000). Muitas metapopulações podem estar ocupando paisagens recentemente fragmentadas, para as quais suas estratégias de dispersão não são evolutivamente adequadas. 


\section{RUPTURA DE ESTRUTURA SOCIAL}

Existe uma outra causa de instabilidade que raramente é considerada em AVPs, e pode não ser completamente reconhecida em planos de conservação: em baixas densidades populacionais, os sistemas sociais de várias espécies pode ser rompidos. A ruptura de estrutura social pode ocorrer por razões que vão de óbvias a sutis. Por exemplo, em densidades baixas, indivíduos provavelmente não encontrarão parceiros em potencial quando estão aptos a reproduzir (e.g. Menges 2000). Mesmo que parceiros potenciais estejam disponíveis, tamanhos populacionais pequenos permitem pouca oportunidade de escolha de parceiros. Este problema pode ser exacerbado se os parceiros potenciais forem parentes próximos. Um experimento demonstrou que dada a escolha entre fêmeas não aparentadas e aparentadas, machos de Peromyscus polionotus preferiram fêmeas não-aparentadas (Ryan \& Altmann 2001, Ryan \& Lacy 2003). Ao comparar eventos reprodutivos de macho com fêmeas aparentadas e nãoaparentadas, foi demonstrado que a reprodução era atrasada e que os tamanhos de ninhada eram menores quando as parceiras disponíveis eram aquelas menos preferidas (Ryan \& Altmann 2001, Ryan \& Lacy 2003). Em várias espécies, observa-se que indivíduos tentam evitar cruzamentos entre parentes (Keane 1990). Evitar a endogamia em populações pequenas pode levar a insucessos frequentes em localizar qualquer parceiro adequado. É possível que o comportamento de escolha de parceiro que tenha evoluído para prevenir endogamia, agora afete a reprodução quando a população é pequena.

Espécies com sistemas sociais complexos podem ser particularmente vulneráveis à problemas resultantes de baixas densidades populacionais. Um exemplo é que para espécies com reprodução cooperativa, o sucesso reprodutivo está fortemente associado ao número de ajudantes (Brito et al. 2004). Pares reprodutores apresentam sucesso reprodutivo baixo a menos que um número mínimo de ajudantes esteja presente, dividindo o esforço de defesa contra predadores de ninho e de provimento das necessidades dos filhotes (Brito et al. 2004). Por exemplo, a ave Campylorhynchus nuchalis possui baixo sucesso reprodutivo a menos que hajam no mínimo dois ajudantes presentes (Rabenold 1990). Se tal população declinar, o recrutamento pode parar quando houverem poucos indivíduos para atuar como ajudantes (Brito et al. 2004). Na verdade, uma população foi resgatada quando imigrantes de uma população vizinha se estabeleceram junto aos grupos reprodutivos restantes (Rabenold et al. 1991). Presumivelmente, a extirpação local desta população teria ocorrido se esta população próxima não tivesse contribuído com indivíduos emigrantes. Modelos baseados em indivíduos podem ser trabalhados para representar detalhes específicos de sistemas sociais, e esta abordagem foi usada para se observar como a interação de processos estocásticos e estrutura de matilhas impactam a viabilidade de lobos (Vucetich et al. 1997) e cães selvagens (Vucetich \& Creel 1999).

\section{USOS DA AVP}

A AVP é uma ferramenta flexível, e existem muitas abordagens para se predizer o estado futuro de populações. Elas podem ser agrupadas em abordagens de avaliação (nas quais o objetivo é simplesmente se perguntar o quão bem uma população está ou estará frente a determinada gama de condições) e de manejo (nas quais o objetivo é determinar que intervenções vão reduzir a probabilidade de extinção de uma população) (Morris \& Doak 2002). Uma breve lista de usos possíveis para a AVP se segue (Morris \& Doak 2002), mas devido à alta flexibilidade da ferramenta, esta lista não é completa e rígida, já que pesquisadores podem descobrir novos usos: (1) avaliar o risco de extinção de uma população. Se houver apenas uma população de uma certa espécie, é importante saber se ela pode estar declinando ou não, e portanto se alguma estratégia de manejo deve ser implementada. Saber quando a extinção tem mais chances de ocorrer pode ajudar a determinar a necessidade de intervenção imediata. Mesmo que existam muitas populações, pode ser útil ter uma avaliação quantitativa do status futuro de cada uma; (2) comparar os riscos relativos de duas ou mais populações. Talvez de maior utilidade que uma única medida de risco para uma população é a habilidade de comparar o risco de extinção entre diversas populações da mesma espécie. Tais estimativas comparativas são úties de diversas maneiras, por exemplo, se quisermos saber se toda uma espécie está ameaçada, podemos perguntar se a maioria das suas populações está declinando. Outro exemplo, se nosso objetivo é preservar todas as 
populações de uma determinada espécie, mas temos limitações (financeiras ou de recursos humanos), tais medidas relativas de risco nos permitiriam priorizar quais populações deveriam receber atenção de manejo primeiro. Um último exemplo, se já foi determinado que não é possível preservar todas as populações de uma espécie, métricas de viabilidade relativa podem indicar quais as populações mais promissoras a serem preservadas; (3) analisar e sintetizar dados. Dados de monitoramento são compilados frequentemente para espécies, mas infelizmente planos cuidadosamente considerados para analisar tais dados de tal forma que eles possam ser úteis em direcionar e informar decisões de manejo, são bem menos frequentes. A AVP pode fornecer uma forma de combinar diversos dados de monitoramento (e.g. sobrevivência, reprodução, etc) em uma única avaliação do estado da população e estimar como uma mudança em uma população monitorada se traduz em mudanças na sua viabilidade; (4) identificar estratégias-chave de manejo, processos de viabilidade e/ou estágios de vida como alvos de manejo. A AVP também é uma ferramenta poderosa para identificar estratégias efetivas de manejo. Ela pode guiar quais processos populacionais, ameaças, estratégias de manejo ou estágios de vida teriam o maior impacto na viabilidade. Ela também fornece uma forma de avaliar se determinada estratégia de manejo está tendo o efeito desejado; (5) determinar a eficiência de áreas protegidas (ou sistemas de áreas protegidas) em alcançar determinado nível desejado de proteção. Para qualquer espécie-alvo, o atual número e tamanho de populações tem forte efeito no seu risco de extinção. Um maior número de populações, e de maior tamanho, requerem mais espaço. Se pudermos estimar a quantidade de espaço que cada indivíduo necessita, podemos usar a AVP para estimar o quão grande uma área protegida precisa ser para apresentar uma baixa probabilidade de extinção. Também podemos guiar o design de sistemas de áreas protegidas, a fim de termos um arranjo que maximize a persistência; (6) Determinar quantos indivíduos são necessários para estabelecer uma nova população. Translocações em reintroduções de indivíduos selvagens ou de cativeiro para locais onde a espécie estava originalmente presente, mas de onde foi extirpada, são estratégias geralmente usadas para fundar novas populações. Tais programas enfrentam uma dúvida inevitável: dadas as limitações no número de indivíduos que podem ser gerados e programas de cativeiro, ou serem seguramente removidos de populações existentes, soltar mais indivíduos em determinado local reduz o número de populações que podem ser estabelecidas. A AVP pode ajudar neste caso, fornecendo estimativas de quão pequenas as populações de determinada espécie podem ser e ainda manter uma chance razoável de se estabelecer com sucesso; (7) Estabelecer limites para a exploração de uma população que seja compatível com sua persistência. Diversas espécies apresentam preocupação quanto à sua viabilidade pois são diretamente usadas pela população humana, deprimindo suas taxas de crescimento e seus tamanhos populacionais. Quanta mortalidade adicional de origem antrópica é compatível com a existência continuada da espécie (ou população)? A AVP pode ajudar nesta questão ao modelar os impactos da retirada de indivíduos da população e estimando tais impactos na sua persistência; (8) determinar quantas (e quais) populações são necessárias para se atingir uma probabilidade de persistência desejada. Estimativas de risco para populações separadas podem ser combinadas para se calcular a probabilidade de que pelo menos uma população ainda vá existir em determinada escala de tempo. Desta forma, é possível estimar o quanto de segurança da extinção uma espécie ganharia se preservarmos números e combinações particulares de populações. Tais análises são importantes tanto para grupos de populações isoladas quanto para metapopulações; e (9) listar espécies como ameaçadas de acordo com os critérios da IUCN (IUCN 2001).

\section{A AVP NO BRASIL}

Comparado a outros países, como Austrália e Estados Unidos, o Brasil ainda está atrasado no desenvolvimento de pesquisas teóricas e empíricas em AVP, e no seu uso como ferramenta para o manejo e conservação da biodiversidade. No Brasil, até o momento, 30 publicações foram dedicadas, ou usaram AVP como um componente principal de suas análises com uma leve tendência de crescimento nos útlimos anos (Figura 1). Entretanto, o número de espécies avaliadas por AVPs ainda é pequeno (15) (Tabela 1), e altamente tendencioso para mamíferos (Figura 2) e para a Mata Atlântica (Figura 4). Se 


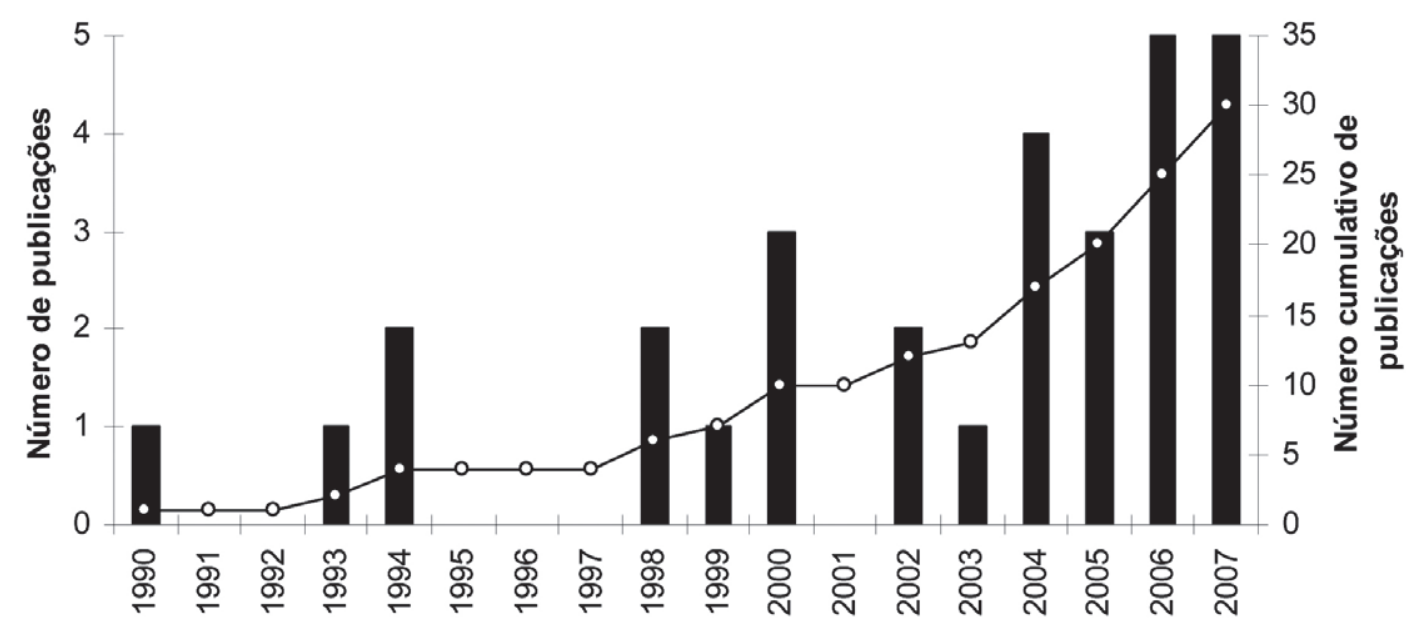

Figura 1. Número absoluto (barras) e cumulativo (círculos) de publicações focadas em Análise de Viabilidade de Populações (AVP) ou usando-a como ferramenta principal, no Brasil.

Figure 1. Absolute number (columns) and cumulative number (circles) of scientific papers either focused on Population Viability Analysis or featuring it as the main analytical tool.

Tabela 1. Uma lista de espécies da fauna brasileira que já foram alvo de estudos de Análise de Viabilidade de Populações (AVP) (apesar de não ter espécies como foco, estudos teóricos são apresentados na lista a seguir).

Table 1. Brazilian species that were targeted by Population Viability Analysis studies. References of theoretical studies are also given, although not focusing on any particular animal species.

\begin{tabular}{l|l}
\hline Espécie & Referência \\
\hline Leontopithecus caissara & Seal et al. (1990), Ballou et al. (1998), Holst et al. (2006) \\
Leontopithecus chrysomelas & Seal et al. (1990), Ballou et al. (1998), Holst et al. (2006) \\
Leontopithecus chrysopygus & Seal et al. (1990), Ballou et al. (1998), Holst et al. (2006) \\
Leontopithecus rosalia & Seal et al. (1990), Kierulff (1993), Ballou et al. (1998), Holst et al. (2006) \\
Brachyteles arachnoides & Rylands et al. (1998) \\
Brachyteles hypoxanthus & Strier (1993/1994, 2000); Rylands et al. (1998), Brito \& Grelle (2006), \\
Coutinho (2007), Brito et al. (in press) \\
Micoureus paraguayanus & Brito \& Fernandez (2000b, 2002), Brito (2002, 2005), Brito \& Grelle (2004), \\
Brito \& Fonseca (2006, 2007) \\
Myrinomys eliasi & Brito \& Figueiredo (2003) \\
Blastocerus dichotomus & Miranda (2004) \\
Tapirus terrestris & Tiepolo et al. (2004) \\
Cavia intermedia & Gatti (2005), Medici et al. (2007) \\
Chrysocyon brachyurus & Oliveira (2006) \\
Aburria jacutinga & Paula et al. (2007) \\
Neothraupis fasciata & São Bernardo (2004) \\
Estudo teórico & Soares (2007) \\
\hline
\end{tabular}

levarmos em conta estimativas que indicam que o Brasil possui aproximadamente 7150 espécies de vertebrados em seu território (Lewinsohn \& Prado 2005), isto significa que apenas $0,2 \%$ da fauna de vertebrados do país já recebeu algum tipo de atenção por estudos de AVP. É interessante notar que alguns trabalhos teóricos sobre AVP também está presente na literatura nacional, indicando que a abordagem de AVP tem sido usada não apenas como ferramenta, mas que pesquisadores brasileiros também estão dedicando pesquisa às questões teóricas envolvendo viabilidade de populações pequenas. Apesar desta tendência ser algo positivo, ainda existem algumas preocupações: (1) a maioria das publicações são 


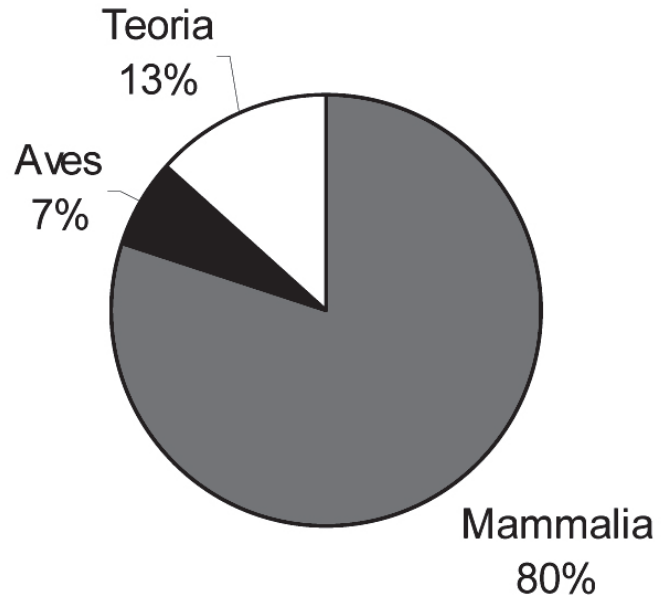

Figura 2. Proporção de publicações empíricas (divididas por grupo taxonômico) e teóricas sobre Análise de Viabilidade de Populações (AVP) no Brasil.

Figure 2. Relative proportion of empirical (presented by taxonomic group studied) and theoretical scientific papers about Population Viability Analysis in Brazil.

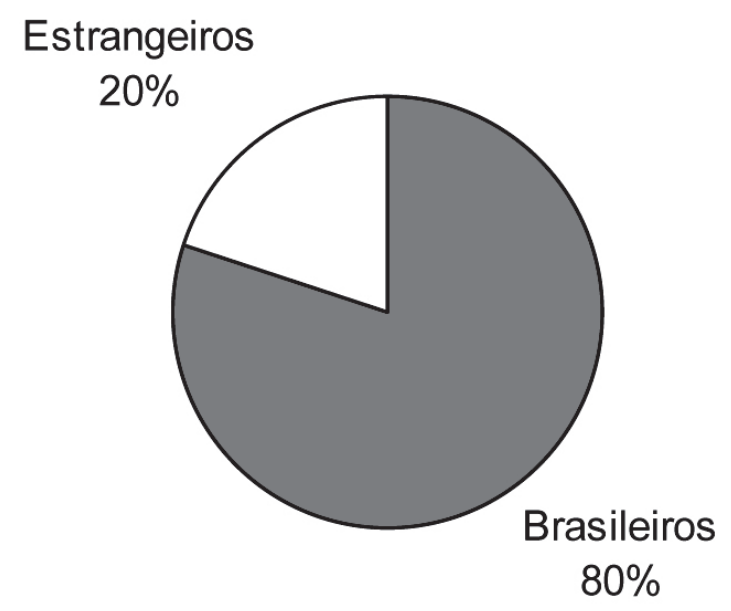

Figura 3. Porcentagem de autores brasileiros e estrangeiros de artigos de Análise de Viabilidade de Populações (AVP) sobre a fauna nacional.

Figure 3. Relative percentage of Brazilian authors among the authors of scientific papers about Population Viability Analysis in Brazil.

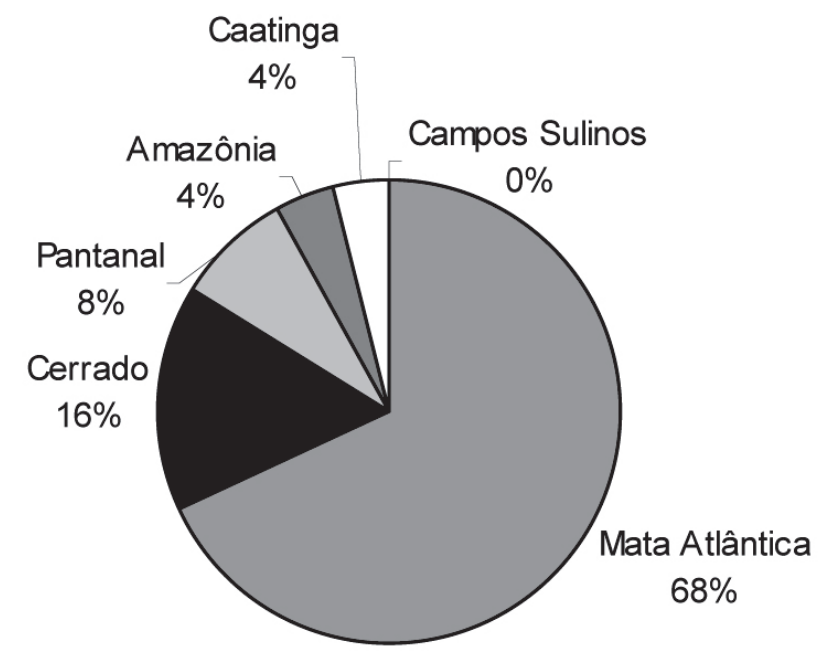

Figura 4. Número de trabalhos de Análise de Viabilidade de Populações (AVP) no Brasil, de acordo com sua cobertura geográfica (por bioma).

Figure 4. Number of scientific papers on Population Viability Analysis in Brazil according with studied biome.

planos de ação (20\%), teses ou dissertações (33\%), sendo consideradas como literatura cinza, de difícil acesso para o leitor; (2) uma porção significativa (20\%) das AVPs publicadas com espécies brasileiras foi conduzida por pesquisadores estrangeiros estudando a biodiversidade nacional (Figura 3). Entretanto o número de autores brasileiros vem crescendo com o tempo; (3) ainda existem tendenciosidades taxonômicas e geográficas nos estudos de AVP no Brasil (Figuras 2 e 4).

Dentre as 15 espécies estudadas no Brasil, apenas seis receberam uma atenção regular ao longo do tempo: as quatro espécies de Leontopithecus, Brachyteles hypoxanthus e Micoureus paraguayanus. As quatro espécies de Leontopithecus foram alvo da primeira AVP dedicada a fauna nacional (Seal et al. 1990). Um plano de ação para recuperar estes primatas altamente ameaçados foi desenvolvido, e a AVP teve um papel fundamental em guiar as estratégias de manejo para o grupo. Um exemplo da natureza dinâmica do processo de AVP, o plano de ação (e a AVP) dos micos-leões vem sendo periodicamente atualizado e monitorado (Seal et al. 1990, Ballou et al. 1997, Holst et al. 2006), e isto resultou na melhoria estado das populações de L rosalia e L. chrysopygus e o sucesso na mudança de seu status de conservação na lista vermelha de espécies 
ameaçadas de extinção da IUCN (de Criticamente em Perigo para Em Perigo). Kierulff (1993) também usou AVP para avaliar ameaças e guiar o manejo de populações de mico-leão dourado na Reserva Biológica de Poço das Antas e arredores, contribuindo para o sucesso na recuperação da espécie. Os muriquis (Brachyteles) também tem sido regularmente foco de AVPs no Brasil (Strier 1993,1994, 2000, Rylands et al. 1998, Brito \& Grelle 2006, Coutinho 2007, Brito et al. 2008), particularmente o muriqui do norte (Brachyteles hypoxanthus). AVPs tem sido usadas para guiar o manejo, avaliar ameaças, estimar PMV, estimar área mínima de habitat e avaliar o papel de sistemas de áreas protegidas em manter populações viáveis da espécie. Um plano de ação também foi desenvolvido para a guiar a recuperação do gênero, e a AVP é uma ferramenta central nesta estratégia (Rylands et al. 1998). O marsupial Micoureus paraguayanus também é uma espécie regularmente alvo de AVPs no Brasil (Brito \& Fernandez 2000b, 2002, Brito 2002, 2005a, Brito \& Grelle 2004, Brito \& Fonseca 2006, 2007), e ilustra a flexibilidade e a ampla gama de cenários nos quais a AVP pode ajudar na conservação da biodiversidade. Similarmente ao muriqui, AVPs foram usadas para guiar o manejo, avaliar ameaças, estimar PMV, estimar área mínima de habitat e avaliar o papel de sistemas de áreas protegidas em manter populações viáveis. Além disso AVPs também foram usadas para avaliar o papel e contribuição relativos de diferentes populações para a dinâmica e viabilidade metapopulacional, e para modelar consequências genéticas e demográficas da fragmentação de habitat e da subdivisão populacional na persistência.

Outras espécies foram estudadas em menor escala. Miranda (2004) estimou a probabilidade de persistência de uma população de tamanduá-bandeira (Myrmecophaga tridactyla) em uma área protegida sob forte pressão antrópica (perda de habitat e incêndios), e a variação de fatores intrínsecos (sobrevivência e reprodução). Gatti (2005) estimou PMVs e modelou impacto do fogo e de diferentes cenários de pressão de caça na persistência de populações de anta (Tapirus terrestris) no estado do Espírito Santo. Recentemente, um plano de ação também foi desenvolvido para preservar e recuperar populações de anta, identificando ameaças e planejando estratégias de manejo, sendo guiado por AVP (Medici et al. 2007). Uma AVP também foi usada para avaliar o impacto da caça ilegal sobre a persistência de uma população insular de jacutingas (Aburria jacutinga) (São Bernardo 2004). Soares (2007) apresentou uma abordagem interessante onde a AVP foi usada para auxiliar o manejo para Neothraupis fasciata, não apenas ranqueando resultados biológicos e de viabilidade, mas incorporando custos econômicos, apresentando um novo fator a ser considerado em AVPs. Brito \& Figueiredo (2003) usaram a AVP para estimar a PMV e a área mínima de habitat adequado e os efeitos da endogamia para o roedor Trinomys eliasi. Mas o mais interessante, a espécie não havia sido avaliada pela IUCN até então, e considerando as categorias e critérios estabelecidos pela IUCN (2001), uma AVP foi usada para avaliar o estado de conservação da espécie, e teve sucesso em provar que a mesma estava ameaçada, sugerindo sua inclusão na lista vermelha mundial de espécies ameaçadas de extinção. Como resultado, a espécie foi avaliada pela Avaliação Global de Mamíferos (Global Mammal Assessment) e foi incluída na lista da IUCN como ameaçada (IUCN 2008). No Brasil, AVPs também foram usadas para estudar ameaças, manejo e viabilidade de Blastocerus dichotomus (Tiepolo et al. 2004), Cavia intermedia (Oliveira 2006) and Chrysocyon brachyurus (Paula et al. 2007). Melhorar a conservação da biodiversidade e as políticas que garantem tal objetivo é, em parte, dependente de se usar os conceitos apropriados e as ferramentas adequadas, que permitem uma identificação precoce dos problemas, uma estimativa acurada da extensão e gravidade destes problemas, a seleção de soluções apropriadas, a implementação com sucesso de tais soluções, e o monitoramento contínuo do problema assim como da efetividade das soluções. A AVP pode auxiliar em todas estas fases do processo de restauração de habitats e de espécies ameaçadas, além de prevenir que espécies que atualmente não estão ameaçadas, entrem nesta categoria. A AVP pode ajudar não apenas pesquisadores brasileiros, mas também tomadores de decisão, em alcançar o objetivo final de avaliar, monitorar e assegurar a viabilidade em longo prazo da biodiversidade do país.

AGRADECIMENTOS: Eu gostaria de agradecer aos organizadores deste número especial, principalmente ao professor Carlos Eduardo V. Grelle pelo convite para submeter este manuscrito e participar da publicação. Também sou grato aos revisores anônimos cujos comentários e sugestões melhoraram a qualidade do presente trabalho. 


\section{REFERENCIAS}

ALLEN, C.R.; PEARLSTINE, L.G. \& KITCHENS W.M. 2001.

Modeling viable mammal populations in gap analysis. Biological Conservation, 99: 135-144.

ALLENDORF, F.W. \& RYMAN, N. 2002. The role of genetics in population viability analysis. Pp 50-85. In: S.B. Beissinger \& D.R. McCullough, (eds.), Population Viability Analysis. University of Chicago Press, Chicago.

BAILLIE, J.E.M.; HILTON-TAYLOR, C. \& STUART, S.N. 2004. A Global Species Assessment. IUCN, Gland. 191p.

BALLOU, J.D. 1997. Ancestral inbreeding only minimally affects inbreeding depression in mammalian populations. Journal of Heredity, 88: 169-178.

BALLOU, J.D.; LACY, R.C.; KLEIMAN, D.; RYLANDS, A. \& ELLIS, S. 1997. Leontopithecus II: The Second Population and Habitat Viability Assessment for Lion Tamarins (Leontopithecus). CBSG, Apple Valley. 148p.

BALMFORD, A.; MACE, G.M. \& GINSBERG, J.R. 1998. The challenges to conservation in a changing world: putting processes on the map. Pp 1-28. In: G.M. Mace, A. Balmford \& J.R. Ginsberg, (eds.). Conservation in a Changing World. Cambridge University Press, Cambridge. 328p.

BEISSINGER, S.R. \& WESTPHAL, M.I. 1998. On the use of demographic models of population viability in endangered species management. Journal of Wildlife Management, 62: 821-841.

BELOVSKY, G.E. 1987. Extinction models and mammalian persistence. Pp. 35-57. In: M.E. Soulé (ed.). Viable Populations for Conservation. Cambridge University Press, Cambridge. 204p.

BOYCE, M.S. 1992. Population viability analysis. Annual Review of Ecology and Systematics, 23: 481-506.

BRITO, D. 2002. Conservação e Manejo do Marsupial Micoureus demerarae em Fragmentos de Mata Atlântica. Dissertação de Mestrado, Programa de Pós-Graduação em Ecologia da Universidade Federal do Rio de Janeiro, Rio de Janeiro, Brasil, 70p.

BRITO, D. 2005a. Análise de Viabilidade de Populações como Ferramenta de Manejo para o Marsupial Micoureus paraguayanus. Tese de Doutorado, Programa de Pós-Graduação em Ecologia da Universidade Federal do Rio de Janeiro, Rio de Janeiro, Brasil, 117p

BRITO, D. 2005b. The importance of sound biological information and theory for ecological economics studies valuing Brazilian biodiversity: a response to Mendonça et al. (2005). Ecological Economics, 55: 5-10.
BRITO, D. \& FERNANDEZ, F.A.S. 2000a. Dealing with extinction is forever: understanding the risks faced by small populations. Journal of the Brazilian Association for the Advancement of Science, 52: 161-170.

BRITO, D. \& FERNANDEZ, F.A.S. 2000b. Metapopulation viability of the marsupial Micoureus demerarae in small Atlantic Forest fragments in south-eastern Brazil. Animal Conservation, 3: 201-209.

BRITO, D. \& FERNANDEZ, F.A.S. 2002. Patch relative importance to metapopulation viability: the Neotropical marsupial Micoureus demerarae as a case study. Animal Conservation, 5: 45-51.

BRITO, D. \& FIGUEIREDO, M.S.L. 2003. Minimum viable population and conservation status of the Atlantic Forest spiny rat Trinomys eliasi. Biological Conservation, 113: 153-158.

BRITO, D. \& da FONSECA, G.A.B. 2006. Evaluation of minimum viable population size and conservation status of the long-furred woolly mouse opossum Micoureus paraguayanus: an endemic marsupial of the Atlantic Forest. Biodiversity and Conservation, 15: 1713-1728.

BRITO, D. \& da FONSECA, G.A.B. 2007. Demographic consequences of population subdivision on the long-furred woolly mouse opossum (Micoureus paraguayanus) from the Atlantic Forest. Acta Oecologica, 31: 60-68.

BRITO, D. \& GRELLE, C.E.V. 2004. Effectiveness of a reserve network for the conservation of the endemic marsupial Micoureus travassosi in Atlantic Forest remnants in southeastern Brazil. Biodiversity and Conservation, 13: 2519-2536.

BRITO, D. \& GRELLE, C.E.V. 2006. Estimating minimum area of suitable habitat and viable population size for the northern muriqui (Brachyteles hypoxanthus). Biodiversity and Conservation, 15: 4197-4210.

BRITO, D.; ROCHA, F.S.; OLIVEIRA, L.C. \& CARVALHO, F.M.V. 2004. Reprodução cooperativa: ajudar ou não ajudar, eis a questão. Bios, 12: 11-24.

BRITO, D.; GRELLE, C.E.V. \& BOUBLI, J.P. 2008. Is the Atlantic Forest protected area network efficient in maintaining viable populations of Brachyteles hypoxanthus? Biodiversity and Conservation, 17: 3255-3268.

BROOK, B.W.; CANNON, J.R.; LACY, R.C.; MIRANDE, C. \& FRANKHAM, R. 1999. Comparison of the population viability analysis packages GAPPS, INMAT, RAMAS and VORTEX for the whooping crane (Grus americana). Animal Conservation, 2: 23-31. 
BROOK, B.W.; O'GRADY, J.J.; CHAPMAN, A.P.; BURGMAN, M.A.; AKÇAKAYA, H.R. \& FRANKHAM, R. 2000. Predictive accuracy of population viability analysis in conservation biology. Nature 404: 385-387.

BROOK, B.W., BURGMAN, M.A., AKÇAKAYA, H.R., O'GRADY, J.J. \& FRANKHAM, R. 2002. Critiques of PVA ask the wrong questions: throwing the heuristic baby out with the numerical bath water. Conservation Biology 16: 262-263.

BROWN, J.H. \& KODRICK-BROWN, A. 1977. Turnover rates in insular biogeography: effect of immigration on extinction. Ecology, 58: 445-449.

BURGMAN, M.A.; FERSON, S. \& AKÇAKAYA, H.R. 1993. Risk Assessment in Conservation Biology. New York: Chapman and Hall. 328p.

CAUGHLEY, G. 1994. Directions in conservation biology. Journal of Animal Ecology, 63: 215-244.

CAUGHLEY, G. \& GUNN, A. 1996. Conservation Biology in Theory and Practice. Blackwell, Oxford. 459p.

CBSG. 2009. CBSG Workshop Reports. www.cbsg.org (acesso em 10/08/2009)

CHARLESWORTH, D. \& CHARLESWORTH, B. 1987. Inbreeding depression and its evolutionary consequences. Annual Review of Ecology and Systematics, 18: 237-268.

CLARK, T.W.; BACKHOUSE, G.N. \& LACY, R.C. 1991. Report of a workshop on population viability assessment as a tool for threatened species management and conservation. Ausralian Zoologist, 27: 28-35.

CLINCHY, M. 1997. Does immigration rescue populations from extinction? Implications regarding movement corridors and the conservation of mammals. Oikos, 80: 618-622.

CONWAY, W. 1995. Altered states: population viability analysis, planning, and caring for wildlife in parks. Pp: 13-19. In: Ballou J.D., Gilpin M. \& Foose T.J. (eds.). Population Management for Survival and Recovery: Analytical Methods and Strategies in Small Population Conservation. Columbia University Press, New York. 376p.

COULSON, T.; MACE, G.M.; HUDSON, E. \& POSSINGHAM, H.P. 2001. The use and abuse of population viability analysis. Trends in Ecology and Evolution 16: 219-221.

COUTINHO, B.R. 2007. Análise de viabilidade populacional do muriqui, Brachyteles hypoxanthus (Primates: Atelidae), em fragmentos de Mata Atlântica de Santa Maria de Jatibá, Espírito Santo. Dissertação de Mestrado. Programa de Pós-Graduação em Biologia Animal da Universidade Federal do Espírito Santo, Espírito Santo, Brasil. 41p.
DE MARCO Jr, P. 1999. Considerações sobre um mamífero esférico: modelos de simulação baseados no indivíduo aplicados a dinâmica de metapopulações. Tese de Doutorado. Programa de Pós-Graduação em Ecologia da Universidade Estadual de Campinas. Campinas, Brasil. 130p.

DIAMOND, J.M. 1975. The island dilemma: lessons of modern biogeographic study for the design of natural reserves. Biological Conservation, 7: 129-146.

ELLNER, S.P., FIEBERG, J., LUDWIG, D. \& WILCOX, C. 2002. Precision of population viability analysis. Conservation Biology 16: 258-261.

FAHRIG, L. \& MERRIAM, G. 1985. Habitat patch connectivity and population survival. Ecology, 66: 1762-1768.

FAHRIG, L. \& MERRIAM, G. 1994. Conservation of fragmented populations. Conservation Biology, 8: 50-59.

FRANKEL, O.H. \& SOULÉ, M.E. 1981. Conservation and Evolution. Cambridge University Press, Cambridge. 366p.

FRANKHAM, R. 1995. Inbreeding and extinction: a threshold effect. Conservation Biology, 9: 792-799.

FRANKLIN, I.R. 1980. Evolutionary change in small populations. Pp. 135-149. In: M.E. Soulé \& B. Wilcox (eds.). Conservation Biology: An Evolutionary-Ecological Perspective. Sinauer Associates, Sunderland. 395p.

GATTI, A. 2005. Análise de Viabilidade Populacional da anta Tapirus terrestris (Perissodactyla; Tapiridae) na Mata Atlântica. Dissertação de Mesrado. Programa de Pós-Graduação em Biologia Anmal da Universidade Federal do Espírito Santo, Espírito Santo, Brasil. 127p.

GILPIN, M. 1996. Forty-eight parrots and the origins of population viability analysis. Conservation Biology, 10: 1491-1493.

GILPIN, M.E. \& SOULÉ, M.E. 1986. Minimum viable populations: processes of extinction. Pp 19-34. In: M.E. Soulé, (ed.). Conservation Biology: The Science of Scarcity and Diversity. Sinauer Associates, Sunderland. 584p.

GOODMAN, D. 1987. The demography of chance extinction. Pp. 11-34. In: M.E. Soulé (ed.). Viable Populations for Conservation. Cambridge Univesity Press, Cambridge. 189p.

GRELLE, C.E.V. \& BRITO, D. 2006. Synergism between gap and MVP analyses. Metodos en Ecologia y Systematica, 1: 16-19.

GYLLENBERG, M. \& HANSKI, I. 1992. Single-species metapopulation dynamics: a structured model. Theoretical Population Biology, 42: 35-61. 
HEDRICK, P.W. 1994. Purging inbreeding depression and the probability of extinction: full-sib mating. Heredity, 73: 363-372.

HEDRICK, P.W.; LACY, R.C.; ALLENDORF, L.W. \& SOULÉ, M.E. 1996. Directions in conservation biology: comments on Caughley. Conservation Biology, 10: 1312-1320.

HOLST, B.; MEDICI, E.P.; MRINHO-FILHO, O.J.; KLEIMAN, D.; LEUS, K.; PISSINATTI, A.; VIVEKANANDA, G.; BALLOU, J.D.; TRAYLOR-HOLZER, B.; RABOY, B.; PASSOS, F.; PARANHOS, K.; VLEESCHOWER, K. \& MONTENEGRO, M.M. 2006. Lion tamarin population and habitat viability assessment workshop 2005, final report. CBSG, Apple Valey. 205p.

IUCN. 2001. IUCN Red List Categories and Criteria Version 3.1. IUCN, Gland. 31p.

IUCN. 2008. 2008 IUCN Red List of Threatened Species. www. redlist.org (acesso em 07/06/2009)

KEANE, B. 1990. The effect of relatedness on reproductive success and mate choice in the white-footed mouse, Peromyscus leucopus. Animal Behaviour, 39: 264-273.

KIERULFF, M.C.M. 1993. Avaliação das populações selvagens de mico-leão-dourado Leontopithecus rosalia e proposta de estratégia para sua conservação. Tese de mestrado. Programa de Pós-Graduação em Ecologia, Conservação e Manejo da Vida Silvestre da Universidade Federal de Minas Gerais, Belo Horizonte, Brasil. 212p.

KINDVALL, O. 1996. Habitat heterogeneity and survival of the bush cricket, Metrioptera bicolor. Ecology, 77: 207-214.

LACAVA, J. \& HUGHES, J. 1984. Determining minimum viable population levels. Wildlife Society Bulletin, 12: 370-376.

LACY, R.C. 1993/1994. What is population (and habitat) viability analysis? Primate Conservation, 14/15: 27-33.

LACY, R.C. 1997. Importance of genetic variation to the viability of mammalian populations. Journal of Mammalogy, 78: 320-335.

LACY, R.C. 2000. Considering threats to the viability of small populations using individual-based models. Ecological Bulletin 48: $39-51$.

LACY, R.C. \& BALLOU, J.D. 1998. Effectiveness of selection in reducing the genetic load in populations of Peromyscus polionotus during generations of inbreeding. Evolution, 52: 900-909.

LACY, R.C.; ALAKS, G. \& WALSH, A. 1996. Hierarchical analysis of inbreeding depression in Peromyscus polionotus. Evolution, 50: 2187-2200.
LANDE, R. 1988. Genetics and demography in biological conservation. Science, 241: 1455-1460.

LANDE, R. 1993. Risks of population extinction from demographic and environmental stochasticity and random catastrophes. American Naturalist, 142: 911-927.

LANDE, R. 1995. Mutation and conservation. Conservation Biology, 9: 782-791.

LANDE, R. 2002. Incorporating stochasticity in population viability analysis. Pp. 18-40. In: S.R. Beissinger \& D.R. McCullough (eds.). Population Viability Analysis. Chicago University Press, Chicago. 593p.

LANDE, R. \& BARROWCLOUGH, G.R. 1987. Effective population size, genetic variation, and their use in population management. Pp. 87-123. In: M.E. Soulé (ed.). Viable Populations for Conservation. Cambridge University Press, Cambridge. 204p.

LEHMKUHL, J.F. 1984. Determining size and dispersion of minimum viable populations for land management planning and species conservation. Environmental Management, 8: 167-176.

LEWINSOHN, T.M. \& PRADO, P.I. 2005. How many species are there in Brazil? Conservation Biology, 19: 619-624.

LINDENMAYER, D.B. \& LACY, R.C. 1995. Metapopulation viability of arboreal marsupials in fragmented old-growth forests: comparison among species. Ecological Applications, 5: 164-182.

LINDENMAYER, D.B. \& POSSINGHAM, H.P. 1994. The Risk of Extinction: Ranking Management Options for Leadbeater's Possum Using Population Viability Analysis. Centre for Resource and Environmental Studies, Australian National University, Canberra. 204p.

LINDENMAYER, D.B.; CLARK, T.W.; LACY, R.C. \& THOMAS, V.C. 1993. Population viability analysis as a tool in wildlife management: a review with reference to Australia. Environmental Management, 17: 745-758.

LYNCH, M. \& LANDE, R. 1998. The critical effective size for a genetically secure population. Animal Conservation, 1: 70-72.

MACARTHUR, R.M. \& WILSON, E.O. 1967. The Theory of Island Biogeography. Princeton University Press, Princeton. 224p.

MEDICI, E.P.; DESBIEZ, A.L.J.; GONÇALVES DA SILVA, A.; JERUSALINSKY, L.; CHASSOT, O.; MONTENEGRO, O.L.; RODRÍGUEZ, J.O.; MENDOZA, A.; QUSE, V.B.; PEDRAZA, C.; GATTI, A.; OLIVEIRA-SANTOS, L.G.R.; TORTATO, M.A.; RAMOS, V.; REIS, M.L.; LANDAU-REMY, G.; TAPIA, A. \& MORAIS, A.A. 2007. Workshop para a conservação da anta brasileira: relatório final. CBSG, Sorocaba. 267p. 
MENGES, E.S. 1986. Predicting the future of rare plant populations: demographic monitoring and modeling. Natural Areas Journal, 6: 13-25.

MENGES, E.S. 2000. Applications of population viability analyses in plant conservation. Ecological Bulletin, 48: 73-84.

MIRANDA, G.H.B. 2004. Ecologia e conservação do tamanduábandeira (Myrmecophaga tridactyla, Linnaeus 1758) no Parque Nacional das Emas. Tese de Doutorado. Programa de Pós-Graduação em Ecologia da Universidade de Brasília, Brasília, Brasil. 81p.

MITTERMEIER, R.A.; GIL P. \& MITTERMEIER C.G. 1997. Megadiversity: Earth's Biologically Wealthiest Nations. CEMEX, Mexico City. 501p.

MORRIS, W.F. \& DOAK, D.F. 2002. Quantitative conservation biology: theory and practice of population viability analysis. Sinauer, Sunderland. 480p.

NORTON, T.W. 1995. Special issue: applications of population viability analysis to biodiversity conservation. Biological Conservation, 73: 91-176.

NUNNEY, L. \& CAMPBELL K.A. 1993. Assessing minimum viable population size: demography meets population genetics. Trends in Ecology and Evolution, 8: 234-239.

OLIVEIRA, C.H.S. 2006. Biologia da conservação na teoria e na prática: o estudo de caso de Cavia intermedia, um dos mamíferos mais raros do planeta. Dissertação de Mestrado. Programa de Pós-Graduação em Ecologia da Universidade Federal do Rio de Janeiro, Rio de Janeiro, Brasil. 93p.

PAULA, R.C.; MEDICI, P. \& MORATO, R.G. 2007. Plano de ação para conservação do lobo-guará: análise de viabilidade populacional e de habitat (PHVA). IBAMA, Brasilia. 160p.

PIMM, S.L. \& BROOKS T.M. 1997. The sixth extinction: How large, how soon, and where? In: P.H. Raven, (ed.). Nature and Human Society: The Quest for a Sustainable World. National Academy Press, Washington. 625p.

PIMM, S.L.; JONES, H.L. \& DIAMOND, J. 1988. On the risk of extinction. American Naturalist, 132: 757-785.

POSSINGHAM, H.P.; LINDENMAYER, D.B. \& NORTON, T.W. 1993. A framework for the improved management of threatened species based on population viability analysis (PVA). Pacific Conservation Biology, 1: 39-45.

POSSINGHAM, H.P., LINDENMAYER, D.B. \& McCARTHY, M.A. 2001. Population viability analysis. Pp 831-843. In: Encyclopedia of Biodiversity Volume 4. Academic Press, New York.
RABENOLD, K.N. 1990. Campylorhynchus wrens: the ecology of delayed dispersal and cooperation in the Venezuelan savanna. Pp. 159-196. In: P.B. Stacey \& W.D. Koenig (eds.). Cooperative Breeding in Birds. Cambridge University Press, Cambridge. 633p.

RABENOLD, P.P.; RABENOLD, K.N.; PIPER, W.H. \& MINCHELLA, D.J. 1991. Density-dependent dispersal in social wrens: genetic analysis using novel matriline markers. Animal Behaviour, 42: 144-146.

RALLS, K. \& TAYLOR, B.L. 1997. How viable is population viability analysis? Pp 228-235. In: Pickett S.T.A., Ostfeld R.S., Shachak M. \& Likens G.E. (eds.). The Ecological Basis of Conservation. Chapman and Hall, New York. 492p.

RALLS, K.; BALLOU, J.D. \& TEMPLETON, A. 1988. Estimates of lethal equivalents and the cost of inbreeding in mammals. Conservation Biology, 2: 185-193.

REED, D.H. \& BRYANT, E.H. 2000. Experimental tests of minimum viable population size. Animal Conservation, 3: 7-14.

REED, J.M., MURPHY, D.D. \& BRUSSARD, P.F. 1998. Efficacy of population viability analysis. Wildlife Society Bulletin 26: $244-251$.

REED, J.M.; DOER, P.D. \& WALTERS, J.R. 1988. Minimum viable population size of the red-cockaded woodpecker. Journal of Wildlife Management, 52: 385-391.

RHYMER, J.M. \& SIMBERLOFF, D. 1996. Extinction by hybridization and introgression. Annual Review of Ecology and Systematics, 27: 83-109.

RONCE, O.; PERRET, F. \& OLIVIERI, I. 2000. Evolutionarily stable dispersal rates do not always increase with local extinction rates. American Naturalist, 155: 485-496.

RYAN, K.K. \& ALTMANN, J. 2001. Selection for male choice based primarily on mate compatibility in the oldfield mouse, Peromyscus polionotus rhoadsi. Behavioral Ecology and Sociobiology, 50: 436-440.

RYAN, K.K. \& LACY, R.C. 2003. Monogamous male mice bias behaviour towards females according to very small differences in kinship. Animal Behaviour, 65: 379-384.

RYLANDS, A.; STRIER, K.; MITTERMEIER, R.; BOROVANSKY, J. \& SEAL, U.S. 1998. Population and habitat viability assessment workshop for the muriqui (Brachyteles arachnoides). CBSG, Apple Valley.

SÃO BERNARDO, C.S. 2004. Abundância, densidade e tamanho populacional de aves e mamíferos cinegéticos no Parque Estadual 
Ilha do Cardoso, SP, Brasil. Dissertação de Mestrado. Programa de Pós-Graduação em Ecologia da Universidade de São Paulo. São Paulo, Brasil. 176p.

SEAL, U.S.; BALLOU, J.D. \& VALLADARES-PÁDUA, C. 1990. Leontopithecus: population viability analysis workshop. CBSG, Apple Valley.

SHAFFER, M.L. 1981. Minimum viable population sizes for species conservation. BioScience, 31: 131-134.

SHAFFER, M.L. 1987. Minimum viable populations: copying with uncertainty. Pp. 69-86. In: M.E. Soulé (ed.). Viable Populations for Conservation. Cambridge Univesity Press, Cambridge. $189 \mathrm{p}$.

SHAFFER, M.L. \& SAMSON, F.B. 1985. Population size and extinction: a note on determining critical population sizes. American Naturalist, 125: 144-152.

SIMBERLOFF, D. 1996. Hybridization between native and introduced wildlife species: importance for conservation. Wildlife Biology, 2: 143-150.

SOARES, C.G.D. 2007. Biologia e conservação de Neothraupis fasciata (Aves: Thraupidae) no Cerrado do Brasil central. Tese de Doutorado. Programa de Pós-Graduação em Ecologia da Universidade de Brasília, Brasília, Brasil. 153p.

SOULÉ, M.E 1980. Thresholds for survival: maintaining fitness and evolutionary potential. Pp: 155-169. In: M.E. Soulé \& B.A. Wilcox (eds.). Conservation Biology: An Evolutionary-Ecological Perspective. Sinauer, Sunderland. 395p.

SOULÉ, M.E. 1985. What is conservation biology? BioScience, 35: 727-734.

SOULÉ, M.E. 1987. Introduction. Pp 1-10. In: M.E. Soulé (ed.) Viable Populations for Conservation. Cambridge Univesity Press, Cambridge. 189p.

STRIER, K.B. 1993/1994. Viability analyses of an isolated population of muriqui monkeys (Brachyteles arachnoides): implications for primate conservation and demography. Primate Conservation, 14/15: 43-52.

STRIER, K.B. 2000. Population viabilities and conservation implications for muriquis (Brachyteles arachnoides) in Brazil's Atlantic Forest. Biotropica, 32: 903-913.

TIEPOLO, L.M.; FERNANDEZ, F.A.S. \& TOMAS, W.M. 2004. Conservation of the marsh deer Blastocerus dichotomus (Illiger, 1815) (Mammalia, Cervidae) in Ilha Grande National Park and its environs (Paraná / Mato Grosso do Sul, Brazil). Natureza \& Conservação 2: 132-142.
VAN VALEN, L. 1973. A new evolutionary law. Evolution Theory, 1: 1-30.

VUCETICH, J.A. \& CREEL, S. 1999. Ecological interaction, social organization, and extinction risk in African wild dogs. Conservation Biology, 13: 1172-1182.

VUCETICH, J.A.; PETERSON, R.O. \& WAITE, T.A. 1997. Effects of social structure and prey dynamics on extinction risk in gray wolves. Conservation Biology, 11: 957-965.

WILSON, E.O. 1988. The current state of biological diversity. Pp 3-18. In: E.O. Wilson \& F.M. Peter, (eds.). Biodiversity. National Academy Press, Washington. 538p.

Submetido em 18/03/2009. Aceito em 22/07/2009. 